\title{
Research on Multi-Objective Parameter Dynamic Optimization Design of HEV Considering Mode Transition
}

\author{
Kang HUANG*,**, Zhiyu TANG*, Mingming QIU*,**, Di WU***, Bingzhan ZHANG*** \\ *Hefei University of Technology, School of Mechanical Engineering, Hefei, China 230009, E-mail: hfutqmm@hfut.edu.cn \\ (Corresponding author), E-mail: 17398378769@163.com \\ **National and Local Joint Engineering Research Center for Automotive Technology and Equipment, Hefei, China \\ 230009,E-mail: Hfhuang98@163.com \\ ***Hefei University of Technology, School of Automotive and Traffic Engineering, Hefei, 230009, China, \\ E-mail: wu85@hfut.edu.cn,E-mail: zhangbingzhan@hfut.edu.cn \\ crossref http://dx.doi.org/10.5755/j02.mech.29243
}

\section{Nomenclature}

EV stands for Electric Vehicle, EMS stands for Energy Management Strategy, HEV stands for Hybrid Electric Vehicle, ICE stands for Internal Combust Engine, MG stands for Motor or Generator, MB stands for Mode boundaries, NVH stands for Noise Vibration and Harshness, ECMS stands for Minimum control strategy of equivalent fuel consumption, PSO stands for particle swarm optimization, SOC stands for State of Charge.

\section{Introduction}

With the deterioration of the environment and the depletion of resources, the development of hybrid electric vehicles is growing. For the optimization of hybrid electric vehicle, researchers have put forward many effective methods [1 - 3]. In terms of optimization objectives, on contrary to the objectives of dynamic performance and travel expenditure $[4-6]$, researchers and manufactures are giving increasing attention to enhance ride comfort and driving enjoyment $[7-8]$, i.e., the travel quality.

\subsection{Optimization of powertrain and EMS}

Researchers used different optimization methods for the vehicle driveline or control strategy to improve the fuel economy, power performance, and comfort [9-11]. Gear ratios were optimized with a cost function of economic performance in [12] for an all-EV. As for HEV, because of the highly intertwined relationship between powertrain configurations and EMS, the driveline optimization is generally carried out with key parameters of the control strategy. In $[13,14]$, in order to improve the efficiency of the optimization process, two traditional optimization algorithms were combined. But because the traditional optimization algorithm is to get only one optimal solution, so the accuracy is not guaranteed. [15] proposed an improved genetic algorithm based on adaptive crossover probability and mutation probability, the algorithm solves the accuracy problem effectively, but the optimization process is complicated. [16] proposed a method to optimize the parameters of ECMS through PSO. These two algorithms can solve the problem of accuracy and efficiency of the optimization algorithm, but because of the high coupling between the powertrain and control strategy of hybrid electric vehicle, it is not a good choice to separate the two parameters. $[4,17,18]$ considering the coupling problem of hybrid electric vehicle, the powertrain parameters and control strategy parameters, such as transmission ratio and EMS threshold, were considered in the optimization process. [19] directly uses deep learning to train energy management strategies so that they have better performance under comprehensive conditions. However, the choice of objective function was still the performance of fuel economy or vehicle emission in traditional optimization.

Automated vehicle is a system concerning not only continuous states but also discrete states, such as modes and engine start-stop in HEV case. Detailed mode-transition strategy for parallel HEV was studied in [20 - 22]. Albeit the compensation method by coordinating the driveline components can smooth the shifting process, the life-spans of the actuators, such as synchronizers and clutches, are sacrificed and trade-offs between dynamic requirement and driving comfort must be made if these transitions frequently occur. [23] added a confirmation layer in the algorithm to ensure that the mode will not switch frequently. However, due to the optimization method, the optimization process may take a lot of time, and the final-result is only an optimal solution with poor accuracy.

The researchers also put forward proposed the $\mathrm{NVH}$ problem of the engine, motor and transmission system in hybrid electric vehicles and the method of suppression. established a hybrid electric vehicle model suitable for power distribution. The control method of motor compensation torque was proposed to compensate the vibration source, and the vibration transmission path was established to change the dynamic characteristics of the engine during the start-stop process. In [24], a mathematical modeling of the vibrations observed at the level of the electromechanical coupling between the internal combustion engine and the generator in the series architecture of HEVs, named (SHEVs), is established using the Lagrangian theory. And conducts nonlinear analysis of undesired vibration of hybrid electric vehicle powertrain. [25] investigates an algorithm that can eliminate torque fluctuations caused by engine start and stop. [26] present a coordinated control strategy considering the different dynamic response characteristics of ICE and MG. [27] added the time-varying trajectory disturbance prediction based on echo state network to the vehicle active vibration control method and realized the active vibration control of hybrid electric vehicle powertrain.

After synthesizing the above research, this paper starts from the following three points to distinguish this research from the previous studies: 1) A new multi-objective 
dynamic optimization algorithm considering mode transition is proposed. The decoupling of powertrain and EMS is completed, and the dynamic cycle optimization of parameters is realized. 2) MB is defined in order to reduce the mode transition frequency and increase driving comfort. 3) The high-efficiency working area of the engine is divided into upper and lower boundaries. The frequent start-stop of the engine is effectively controlled, and the operating points of engine and MGs are ensured to appear in the high-efficiency area as far as possible.

\subsection{Overview}

The structure of the paper is as follows: The section II gives the structure of the vehicle and the main modes of hybrid electric vehicle. The section III defines the objective function of the multi-objective dynamic algorithm and develops the energy management strategy and defines the MB. Determines the optimization parameters and constraints. The section IV gives the simulation results and analysis. Finally, the conclusion is given in section $\mathrm{V}$.

\section{Powertrain construction and driving mode}

\subsection{Vehicle structure and parameters}

This paper selects a multi-mode three gear hybrid powertrain, as shown in Fig.1.

The powertrain adopts dual-clutch $C_{2}$ and $C_{3}$ for power coupling. The engine and MG1 are arranged coaxially, and MG1 is connected with the engine output shaft through $C_{1}$. The dual-clutch is used to realize the arbitrary coupling of three power sources and the change of gear $\left(G_{1}\right.$, $\left.G_{2}, G_{3}\right)$, so as to adapt to the power demand under different working conditions. When the vehicle is braking, the energy recovery of MG1 or MG2 can be realized through the combination of dual-clutches.

With the coordination of clutches, synchronizers and brake, the vehicle structure can realize more additional modes.

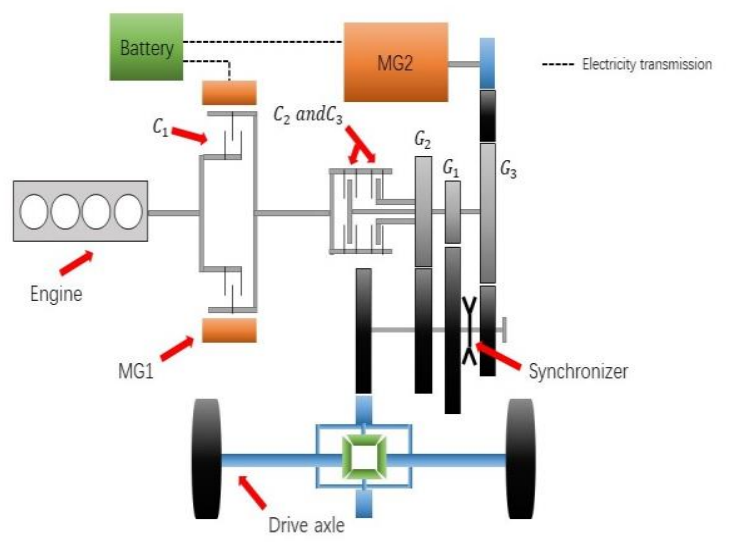

Fig. $1 \mathrm{HEV}$ power-train

\subsection{Vehicle driving mode}

For the multi-mode hybrid electric vehicle mentioned in this paper, its advantage is not only the sufficient power under the guarantee of three power sources, but also that the vehicle can adjust the driving mode according to different working conditions. There are many kinds of modes, up to 10 kinds in total. Because it is impossible to use all modes under ordinary working conditions, the driving charging mode and standstill charging mode are only triggered when the SOC is lower than $30 \%$, and they sacrifice drivability. This paper only gives a brief introduction of the following common modes:

1) Engine separate drive mode. When the vehicle is in high-speed motion and reaches the high efficiency area of the engine, or the torque provided by the MG is insufficient, the engine starts. The clutch $C_{1}$ and $C_{2} / C_{3}$ are combined. The engine power is transmitted to the transmission, and then to the drive axle and wheels through the output shaft.

2) Single motor drive mode. When the vehicle starts or the power demand is low, no need to start the engine, the clutch $C_{1}, C_{2}$ and $C_{3}$ are separated, and the power is directly transmitted to the drive axle and wheels by the MG2 through the transmission.

3) Dual motor drive mode. When the vehicle torque demand is high, but it does not reach the engine operating point, the clutch $C_{2}$ and $C_{3}$ are combined. The power of MG1 is coupled on the transmission shaft through the dual-clutch with MG2, and then transmitted to the drive axle and wheels through the transmission.

4) Engine + single motor hybrid drive mode.In this mode, the engine is always in the high efficiency zone, and MG1 is used as auxiliary speed regulator and generator when necessary, the clutch $C_{1}$ and $C_{2}$ are combined. The engine power and MG2 power are coupled on the input shaft and transmitted to the drive axle and wheels through the transmission.

5) Engine + dual-motor hybrid drive mode. Compared with the engine + single motor hybrid drive mode, clutch $C_{3}$ is combined. Engine power is coupled with MG1 power through $C_{1}$, coupled power is coupled with MG2 power on input shaft again, and finally transmitted to drive axle and wheel through transmission. In this mode, the maximum power output is achieved.

6) Engine charging mode. The vehicle runs at high speed, the engine is at the best economic speed, and control MG2 does not work. Part of the engine power meets the driving power of the vehicle, and the other part of the surplus power drives the MG1 through the clutch $C_{1}$ to charge the power battery.

7) Idle charging mode. When the vehicle stops for a short time, the vehicle is in neutral, and the engine drives the MG1 through the clutch $C_{1}$ to charge the power battery.

8) Braking energy recovery mode. When the vehicle brakes, the wheel power will be transmitted to MG2 through the output shaft and transmission in the opposite direction, driving MG2 to generate electricity and charge the power battery.

Due to the complexity and variety of vehicle modes described in the previous part, vehicles need to face many problems in the process of driving. The hybrid electric vehicle discussed in this paper has three power sources, the complex powertrain is closely related to EMS, which brings the decoupling problem in the optimization process and the complexity of the mode will lead to frequent switching during the operation of the vehicle, reduce the service life of parts, and affect the driving comfort and frequent start-stop of engine is the main source of vibration and shock, and it will also lead to the reduction of fuel economy. 


\section{Problem formulation}

In view of the problems of multi-mode hybrid electric vehicle proposed in the previous chapter, for the problem of highly intertwined relationship between powertrain and EMS, in this chapter, a new multi-objective dynamic optimization algorithm is proposed to realize the decoupling problem of powertrain and control strategy in hybrid electric vehicle. For the characteristics of multi-mode, the mode transition frequency function is added to the objective function. An improved EMS is proposed to effectively control the start-stop of the engine and the efficient operation of the power source.

\subsection{Multi objective dynamic optimization method}

According to the objectives and parameters of this optimization, the mathematical pattern of optimization is as follows:

$$
F_{(x)}=\left[f_{t_{a c c}}, f_{e c o}, f_{f}, f_{s}\right]
$$

where: $f_{t_{a c c}}$ is the dynamic performance function, which is the acceleration time of 100 kilometers; $f_{\text {eco }}$ is the economic performance function, which is fuel consumption and power consumption; $f_{f}$ is the comfort performance function, which is the frequency of mode transition; $f_{s}$ is the smoothness performance function, which is the shift impact degree.

The function in the mathematical pattern involves two aspects of high coupling in hybrid electric vehicle, one is power transmission system, the other is control strategy. For this kind of problem, researchers put forward the corresponding optimization method. However, most of the existing studies focus on the optimization of powertrain parameters or control strategy parameters, and do not consider the highly related problems. The multi-objective dynamic optimization method proposed in this paper.

Considering that the particle swarm optimization algorithm is more suitable for the powertrain mentioned in this paper, based on the particle swarm optimization algorithm, the dynamic optimization algorithm in this paper includes two optimization layers. The first layer is the optimization of economic and dynamic performance, with fuel consumption, power consumption and $100 \mathrm{~km}$ acceleration time as the objective function, and the second layer is the optimization of comfort performance considering mode transition, with mode transition frequency as the objective function, impact is the constraint condition.

The algorithm will output 25 groups of optimization parameters after the first level optimization, and every individual has good performance; in the second layer, every individual is used as the initial value to generate 25 groups of optimization parameters. In this way, the sample size can reach 625 . The optimization results of the second layer will be substituted into the first layer in real time to recalculate the economic and dynamic performance. On the one hand, it can ensure that the number of samples is large enough, on the other hand, the accuracy of the optimized final-results can also be guaranteed.

The basic flow of the algorithm is as follows:

1) The seven optimization parameters obtained by the traditional optimization method is taken as the initial value.
2) The system randomly generates 25 groups of populations to calculate economic and dynamic performance respectively.

3) Then, the position and speed of individuals in the population are updated continuously to generate a new population.

4) When the number of iterations of the first optimization is reached, 25 groups of data with the best economic and dynamic performance will be automatically selected.

5) After the first level optimization, we get 25 sets of $F_{(1)}$, in which each solution set (seven optimization parameters) is close to $\min F_{(1)}$. Then, these solution sets are substituted into the second level optimization calculation $F_{2}$ to get the solution sets which are close to $\operatorname{minF}_{2}$.

6) The final solution set which is close to $m i n F_{2}$ will return to the first layer for calculation again, in order to realize real-time dynamic optimization.

7) Finally, the four functions are given different weights to obtain the optimal solution. Considering that the function of power performance and ride comfort was taken as the constraint condition in the optimization process, the weight coefficient was selected as 0.9 , and the function of economic performance and comfortable performance as the two aspects with more emphasis was selected as the weight coefficient of 1 . The specific process is shown in the Fig. 2.

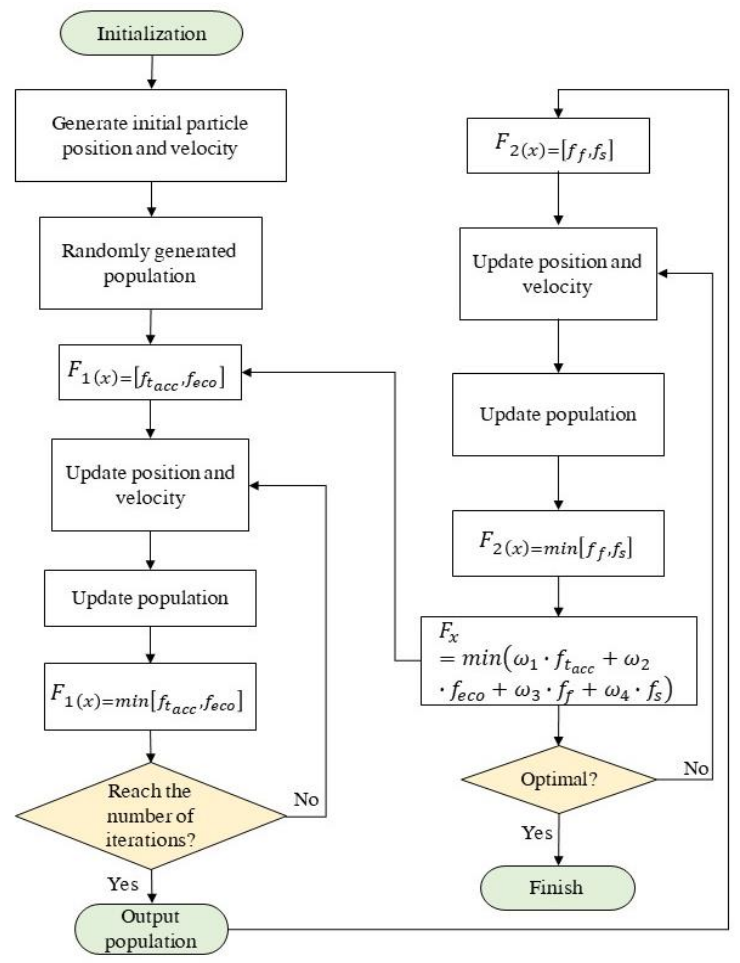

Fig. 2 Optimization flow chart

The transmission system and EMS in hybrid electric vehicle system are complex. The multi-objective dynamic optimization algorithm proposed in this paper can decouple the transmission system and EMS in hybrid electric vehicle.

Different from the traditional method, the optimization results of each layer are retained, and each group of transmission ratio has a corresponding set of control strategy parameters, which makes the optimization process have high fault-tolerant performance and large enough sample size. On the one hand, the calculation results of the second 
layer are weighted to get $F_{(x)}$, on the other hand, it will be automatically substituted into the first layer for re-optimization, it effectively reduces the number of iterations and strives for the optimization time.

Most importantly, the multi-objective dynamic optimization algorithm proposed in this paper considers mode transition at the beginning of parameter design, which reduces the workload of parameter matching and calibration in the later stage, and improves the work efficiency of vehicle improvement in the later stage.

\subsection{Optimization objectives}

In this paper, the modal transition frequency and impact degree in the process of vehicle driving are taken as the optimization objectives, and the economy and dynamic performance of the traditional optimization scheme are considered at the same time.

1) Comfort performance. Unlike traditional hybrid optimization, this paper involves the multimode hybrid cars, because the model is more, so the model boundary is more complex, not simply to consider economy and power performance, [28] proposes a coordinated control algorithm for distributing the torque between the engine and the motor. Its purpose is to calculate the torque distribution according to different modes through coordinated control algorithm, and at the same time, the motor provides the torque required to start the engine to reduce the impact in the process of mode switching. However, due to the delay characteristics of the engine, it is impossible to stop-start immediately. When the mode switching is frequent, it will cause strong oscillator of the engine body and affect the driving comfort; on the other hand, the complex mode leads to the calculation load of the algorithm is too large, the torque distribution of the current mode has just been calculated, and the vehicle has entered the next mode. In order to avoid a series of problems caused by engine delay, this paper is to fundamentally reduce the frequency of mode transition and minimize the torque fluctuation and impact in the driving process. The mode transition frequency can intuitively reflect the comfort level, so this paper proposes a comfort objective function:

$$
f_{f}\left(k_{i}\right)=\frac{\operatorname{num}\left(k_{1}, k_{2}, k_{3}\right)}{t},
$$

where: $f_{f}\left(k_{i}\right)$ is the objective function of mode transition frequency; $\operatorname{num}\left(k_{1}, k_{2}, k_{3}\right)$ is the number of mode transition. Sampling is conducted for the mode of a NEDC operating cycle with a sampling interval of $1 \mathrm{~s}$. By comparing the mode data of the two sampling points before and after, if it is the same, it means no switching; if it is different, it means switching the mode once.

2) Smoothness performance. According to the calculation formula of shifting impact, the more gears in a certain range, the less impact the vehicle will have during shifting, and the better ride comfort. As the vehicle structure mentioned in this paper is a three-speed hybrid electric vehicle, because there are few gears, it is necessary to take the impact degree as the constraint condition of ride comfort, optimize the three-speed transmission ratio, and reduce the impact in the process of gear switching as much as possible. In this paper, the impact degree is used to evaluate the ride smooth of vehicles. Meanwhile, the positive impact degree and the negative impact degree are distinguished. The average values of the two are respectively taken as the ride smooth objective function:

$$
f_{s}=\frac{\sum_{i=1}^{n}\left(\frac{i_{g f} i_{g} \eta}{\delta M_{0} r} \cdot \frac{d T_{c}}{d t}\right)}{n},
$$

where: $n$ is the number of sampling points of a NEDC cycle, with $1 \mathrm{~s}$ interval, so $\mathrm{n}$ is $1180 ; i_{g f}$ is final drive ratio; $i_{g}$ is gear transmission ratio; $\eta$ is transmission efficiency; $\delta$ is the rotation mass conversion coefficient; $M_{0}$ is vehicle mass; $r$ is the radius of tire; $T_{c}$ is the actual transmission of torque by the clutch.

3) Dynamic performance. Dynamic performance is assessed by acceleration time of $0-100 \mathrm{~km} / \mathrm{h} t_{a c c}$. Dynamic shifting schedule is designed and deployed.

4) Economic performance. In this paper, the influence of temperature on the battery is not considered, so the conversion between fuel consumption and power consumption is not carried out. Instead, the sum of the price of fuel consumption and power consumption is directly adopted as the economic objective function as follows:

$$
f_{\text {eco }}=p_{\text {fuel }} f_{\text {fuel }}+p_{\text {ele }} f_{\text {ele }} \text {, }
$$

where: $p_{\text {fuel }}$ and $p_{\text {ele }}$ are oil prices and electricity prices; $f_{\text {fuel }}$ and $f_{\text {ele }}$ are respectively fuel consumption and power consumption under NEDC cycle.

This section defines four kinds of objective functions. In the optimization process, the dynamic objective function, the economic objective function and the comfort objective function are taken as the optimization objective function, and the smoothness objective function is taken as the constraint, so that the final result can guarantee excellent performance in four aspects.

The selected transmission ratios of 3 gears are respectively $i_{g 1}, i_{g 2}, i_{g 3}$, final drive ratio $i_{g f}$, setting parameter $k_{1}$ for the boundary between electric mode and hybrid mode, upper and lower limits $k_{2}, k_{3}$ of engine efficiency curve. A total of 7 parameters are used as optimization parameters. Need of special note is that $k_{1}$ is the start-stop line of the engine in hybrid mode, which is related to the frequent startstop of the engine. $k_{2}$ and $k_{3}$ are efficient engine working curve of upper and lower, it is used to control the engine operating points as far away as possible from the boundary near, on the one hand, due to the engine efficiently workspace is approximate elliptic irregular scope, this limitation does not affect the engine working in high efficient area; On the other hand, reducing the distribution of working points at the boundary can effectively reduce frequent mode transition during vehicle driving, especially the multi-mode hybrid electric vehicle in this paper. At the same time, reducing the start and stop of engine or motor can also effectively control vehicle comfort. The 7 parameters represent driveline parameters and control strategy parameters, which are connected through the vehicle model, and four objective functions are generated after the simulation of driving conditions.

\subsection{Logical threshold energy management strategy}

Due to the diversity of vehicle modes, the logic 
threshold energy management strategy becomes complicated accordingly. The energy management strategy selected in this paper takes into account the efficient engine working area and demarcates the upper and lower limits of engine mode boundary to prevent the frequent transition of modes. The energy management strategy as shown in the Fig. 4.

Table 1.
Detailed description of each mode as shown in the

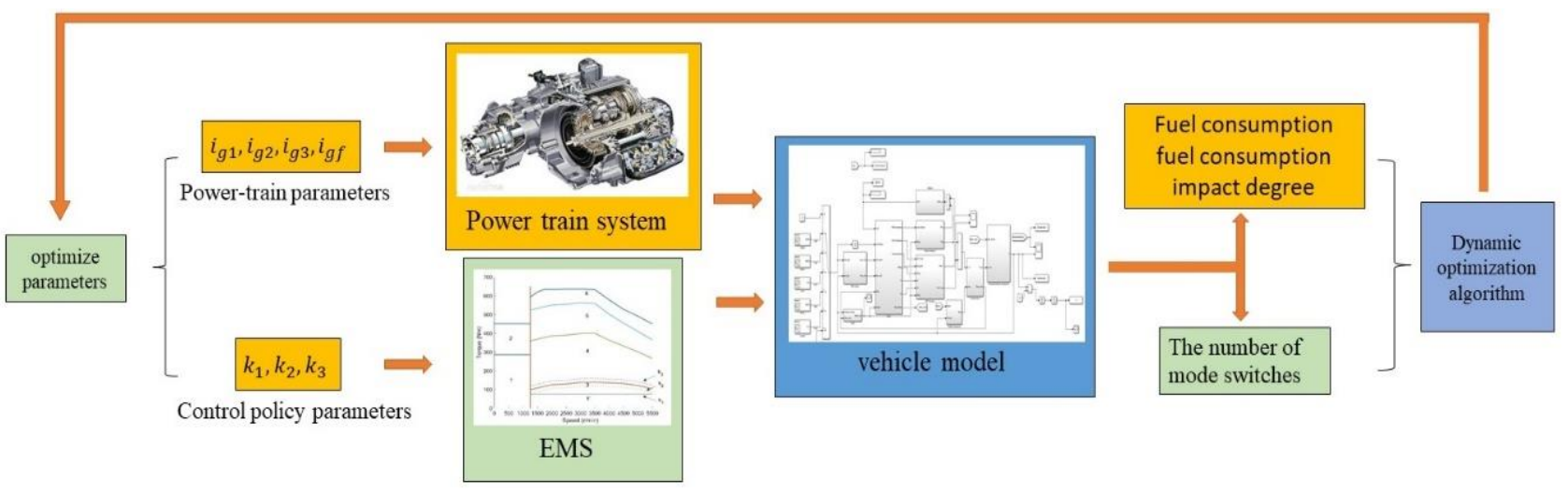

Fig. 3 Relationship between parameters and objective function

Table 1

Logical threshold energy management strategy

\begin{tabular}{|c|c|c|}
\hline HEV mode & Mark & Operating condition \\
\hline Mode 1 & OEM & Single motor operation \\
\hline Mode 2 & TEM & Double motor operation \\
\hline Mode 3 & ENA & Engine in efficient working area \\
\hline Mode 4 & EM1S & Engine in efficient working area, motor 2 provides insufficient torque \\
\hline Mode 5 & EM12S & Engine in efficient working area, motor1 and motor 2 provide insufficient torque \\
\hline Mode 6 & FUL & Engine is not in the optimal working area, motor 1 is in parallel with motor 2 \\
\hline Mode 7 & BRA & Motor 2 for energy recovery \\
\hline
\end{tabular}

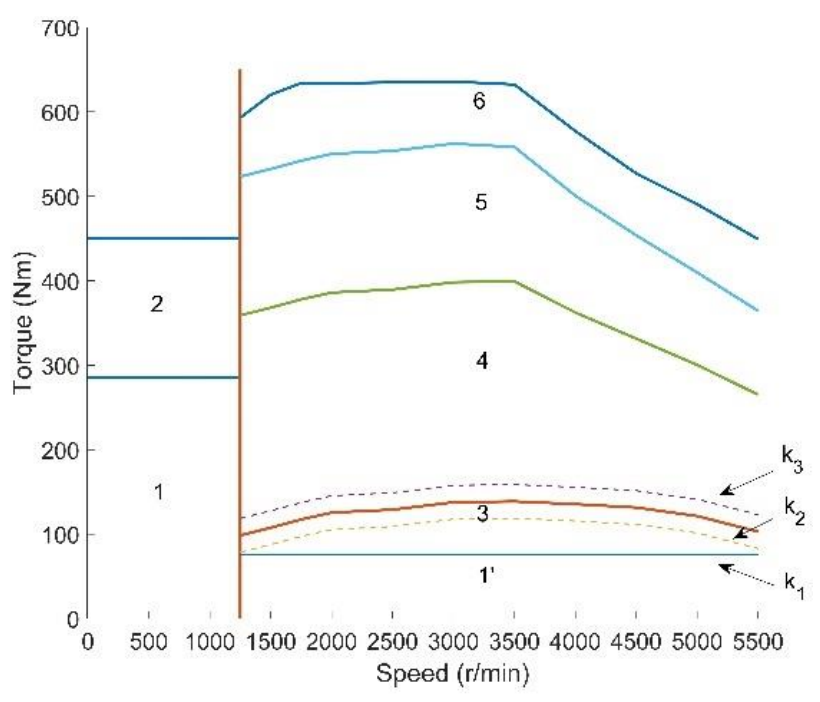

Fig. 4 Energy management strategy

The $\mathrm{x}$-coordinate in the figure represents the rotation speed on the coupling axis, and the y-coordinate represents the torque after coupling. The numbers 1-6 correspond to the patterns 1-6 in Table 2 . The vertical dividing line represents the engine's idle speed, the left represents pure electric mode, and the right, from bottom to top represent engine starting boundary $k_{1}$ (the dividing line between 1 ' and 3 ), engine efficiency curve (the dividing line between 3 and 4), coupling boundary between engine and MG2 (the dividing line between 4 and 5), coupling boundary between engine and two motors (the dividing line between 5 and 6) and the coupling curve of engine external characteristic curve with MG1 and MG2 external characteristic curve.

\subsection{Constraints for the vehicle design}

1) Maximum vehicle velocity. To reach the maximum velocity, the following constraints must be satisfied:

$$
i_{g 3} i_{g f} \leq \frac{0.377 n_{\max } r}{v_{\max }}
$$

where: $v_{\max }$ is the requested maximum velocity of the vehicle; $n_{\max }$ is the maximum speed of the MG2; $r$ is the radius of tire.

$$
\begin{gathered}
\text { 2) Minimum gradeability: } \\
i_{g 1} i_{g f} \geq \frac{M_{0} g(f \cos \alpha+\sin \alpha) r}{T_{\max } \eta}
\end{gathered}
$$

where: $M_{0}$ is the vehicle mass; $f$ is the friction coefficient; $\alpha$ is the grade angle; $g$ is gravity acceleration; $\eta$ is the overall efficiency; $T_{\max }$ is the peak torque of the powertrain.

3) The relationship of gear ratio. For smoother shifting, the constraints can be described as:

$$
\begin{aligned}
& 1.3<\frac{i_{g 1}}{i_{g 2}}<1.8, \\
& 1.3<\frac{i_{g 2}}{i_{g 3}}<1.8 .
\end{aligned}
$$

4) Model boundary. The value of model boundary $k_{1}$ is sure to be within the motor torque range of $5500 \mathrm{r} / \mathrm{min}$, because it involves the engine starting, which will bring a greater degree of influence. Therefore, when the engine 
torque is taken as the input load, the engine is started by two motors, and the residual torque motor is maintained at the same time. In addition, the value of $k_{2}$ and $k_{3}$ should not be too large, otherwise the engine cannot be guaranteed in the high efficiency range. The selected optimization parameters shown in the Table 2.

Table 2

Parameter optimization range

\begin{tabular}{|c|c|c|c|}
\hline Parameter & Definition & Initial value & Range \\
\hline$i_{g 1}$ & First gear ratio & 2.1856 & $2 \sim 3.8$ \\
\hline$i_{g 2}$ & Second gear ratio & 1.32 & $0.8 \sim 1.6$ \\
\hline$i_{g 3}$ & Third gear ratio & 0.64 & $0.6 \sim 1.3$ \\
\hline$i_{g f}$ & Final drive ratio & 4.4 & $4 \sim 5$ \\
\hline$k_{1}$ & Engine start-stop line & 80 & $80 \sim 110$ \\
\hline$k_{2}$ & $\begin{array}{c}\text { Lower limit of engine } \\
\text { optimal curve }\end{array}$ & 0 & $0 \sim 50$ \\
\hline$k_{3}$ & $\begin{array}{c}\text { Upper limit of engine } \\
\text { optimal curve }\end{array}$ & 0 & $0 \sim 50$ \\
\hline
\end{tabular}

Vehicle model is established via Simulink/MATLAB. The pre optimization parameters mentioned in this paper are the results obtained by using the tra- ditional particle swarm optimization algorithm, and are now being used in the enterprise test vehicle. The overview of the vehicle model is shown in Fig. 5. Major parameters are shown in Table 3.

Table 3

Key parameters of the vehicle

\begin{tabular}{|c|c|c|}
\hline Component & Parameter & Value \\
\hline \multirow{4}{*}{ Vehicle } & Mass, $\mathrm{kg}$ & 2100 \\
\cline { 2 - 3 } & Windward area, $\mathrm{m}^{2}$ & 2.56 \\
\cline { 2 - 3 } & Rolling coefficient & 0.01 \\
\cline { 2 - 3 } & Aerodynamic coefficient & 0.36 \\
\cline { 2 - 3 } & Tire radius, $\mathrm{m}$ & 0.353 \\
\hline \multirow{3}{*}{ ICE } & Max power, Kw; & 108, \\
& Speed, $\mathrm{r} / \mathrm{min}$ & 5500 \\
\hline \multirow{2}{*}{ MG1 } & Torque, Nm; & 216, \\
& Speed, $\mathrm{r} / \mathrm{min}$ & 3700 \\
\hline \multirow{2}{*}{ MG2 } & Power, Kw & 55 \\
& Max torque, Nm & 285 \\
\hline \multirow{2}{*}{ Battery } & Power, Kw & 40 \\
\cline { 2 - 3 } & Max torque, Nm & 330 \\
\hline
\end{tabular}

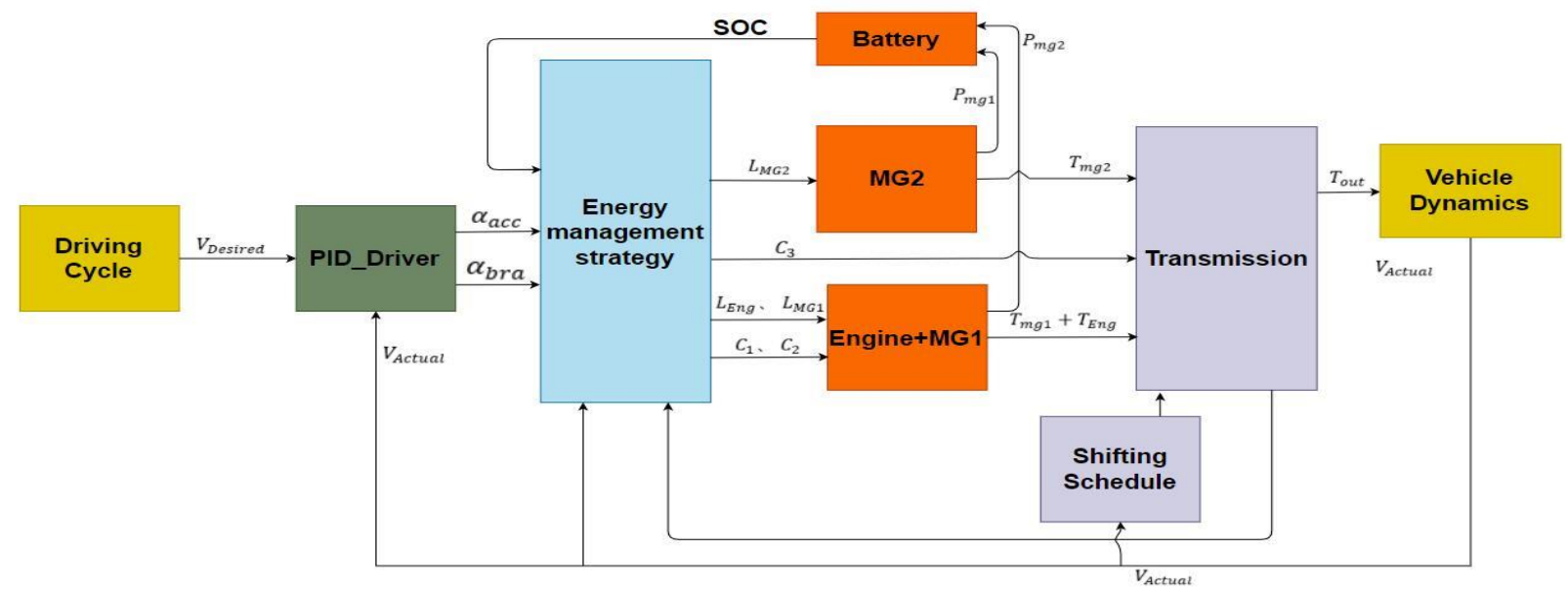

Fig. 5 Vehicle model

\section{Optimization results and analysis}

\subsection{Optimization results of EMS}

In this paper, when we choose the final solution, we give four objective functions corresponding weights $\omega_{1}$, $\omega_{2}, \omega_{3}, \omega_{4}$. Because this paper mainly considers comfort performance and economic performance, the weights are 0.9 , $1,1,0.9$ respectively. Use the following algorithm to find the optimal solution:

$$
F_{x}=\min \left(\omega_{1} \cdot f_{t_{\text {acc }}}+\omega_{2} \cdot f_{\text {eco }}+\omega_{3} \cdot f_{f}+\omega_{4} \cdot f_{s}\right) .
$$

The iteration process is shown in Fig. 6. It can be seen from the figure that the fitness function converges after the 35th generation, because the parameters of the powertrain and the control strategy are optimized synchronously in the optimization algorithm. The output of the first level is a population, and every individual in the population generates its own population in the second level. At the same time, the results of the second layer will return to the first layer to recalculate the objective function, which also makes it unnecessary to iterate too many times.

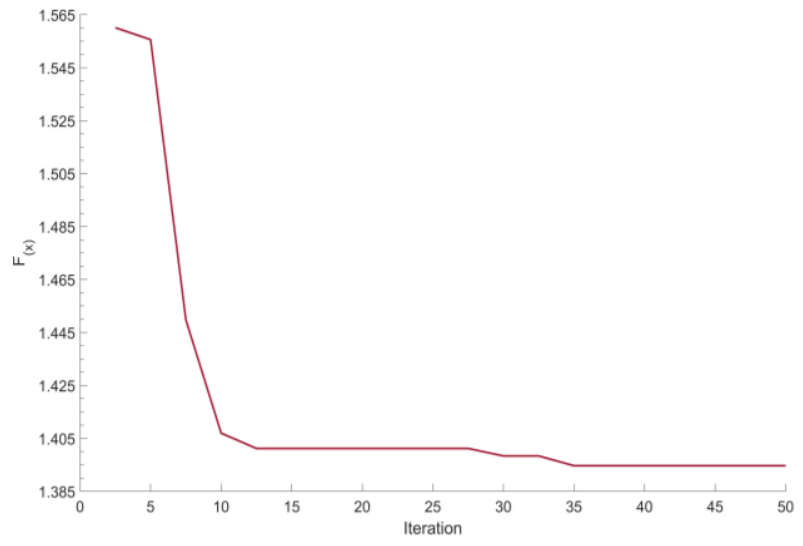

Fig. 6 Iteration process

Since the results of 50 generations of optimization have converged, it is not necessary to use large samples to ensure the accuracy of calculation when selecting the optimization results. In this paper, 25 groups of data in the optimization results are selected, select the best from the best.

From the three-dimensional scatter diagram, we can see that only considering the economic performance and 


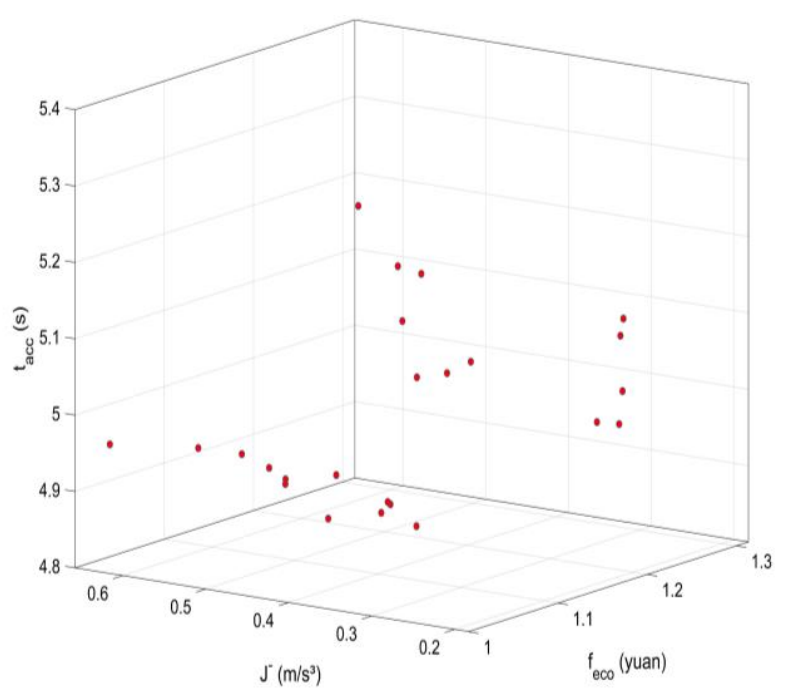

Fig. 7 Scatter distribution

dynamic performance, they are consistent with the traditional dual-objective optimization results, presenting obvious contradictory solutions, and the smoothness performance can play a certain constraint role on them. The Fig. 8 shows the comparison of economy-dynamic performance, economy-smoothness, and power-smoothness scatter distribution results respectively.

The figure shows that economic performance, dynamic performance and smooth performance are contradictory solutions to each other. The comparison result of negative impact degree is similar to that of positive impact de-

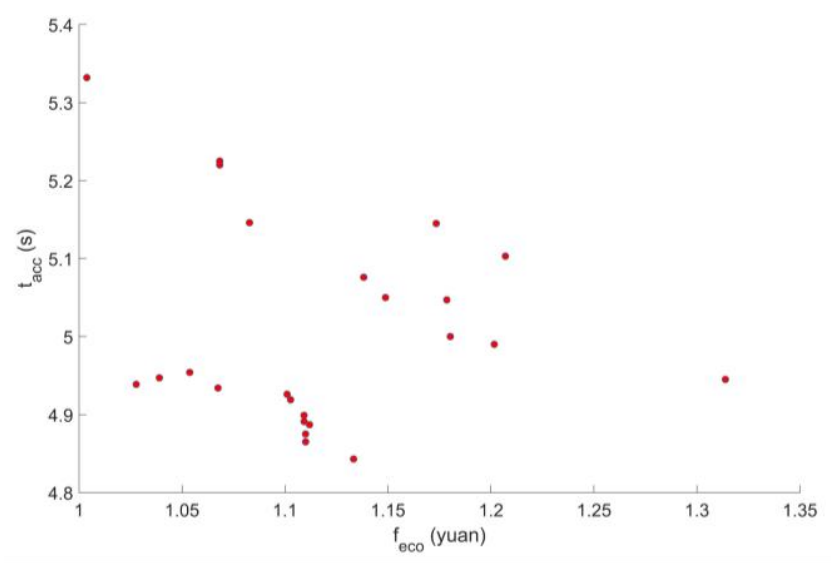

a

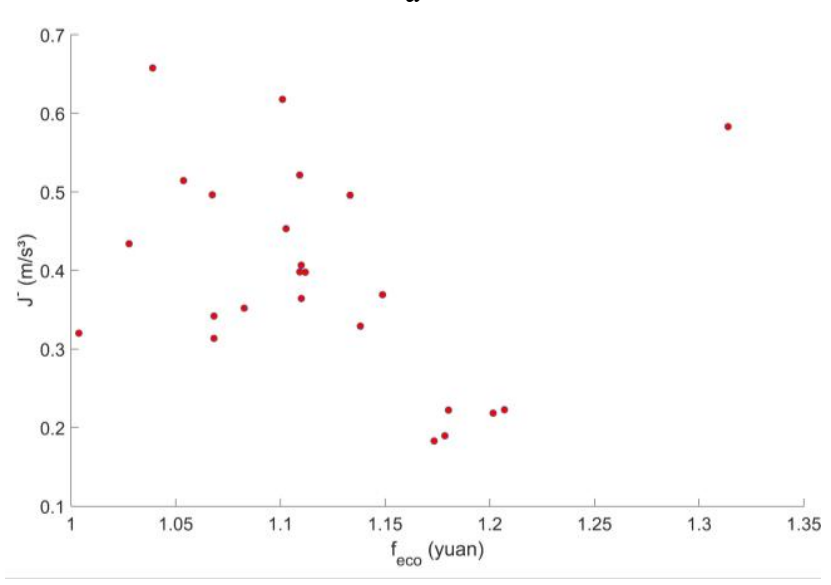

b

Fig. 8 Optimization results of EMS

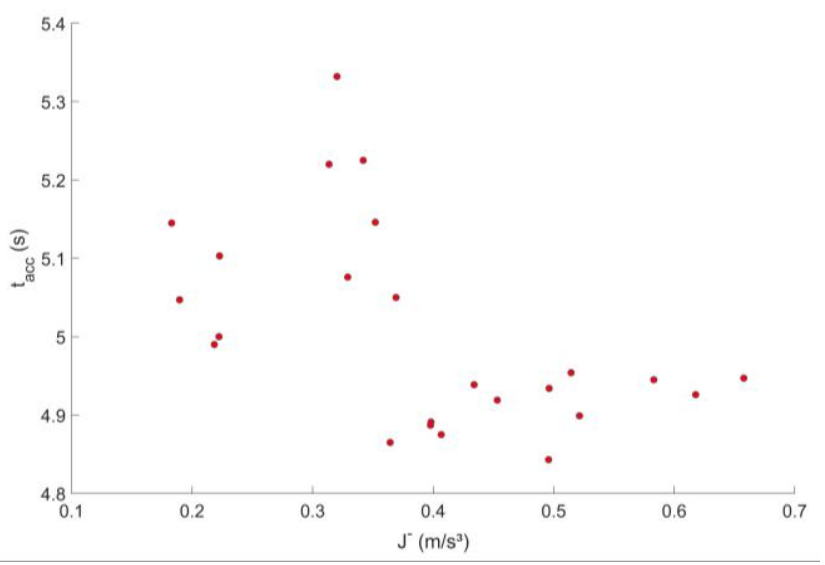

C

Fig. 8 Continuation

gree, so it is no longer given. Since these solutions are feasible, but considering that the dynamic performance should be good and the economic performance and smooth performance should be as good as possible, the final Pareto optimal solutions selected is:

$$
\begin{aligned}
& x=\left(i_{g 1}, i_{g 2}, i_{g 3}, i_{g f}, k_{1}, k_{2}, k_{3}\right)= \\
& =(2.3805,1.1,0.8084,4.7824,107,5.01,39.8927) .
\end{aligned}
$$

\subsection{Results comparison and analysis}

The optimal solution was simulated on the Silulink platform, and the results were compared with traditional optimization, as shown in the Fig. 9.

In this paper, the dynamic performance of the vehicle is evaluated by an acceleration time of 100 kilometers. At the same time, the gear shift plan is used to limit only once gear shift in the acceleration phase. It can be seen from the figure that the optimized acceleration process and acceleration time have been significantly improved.

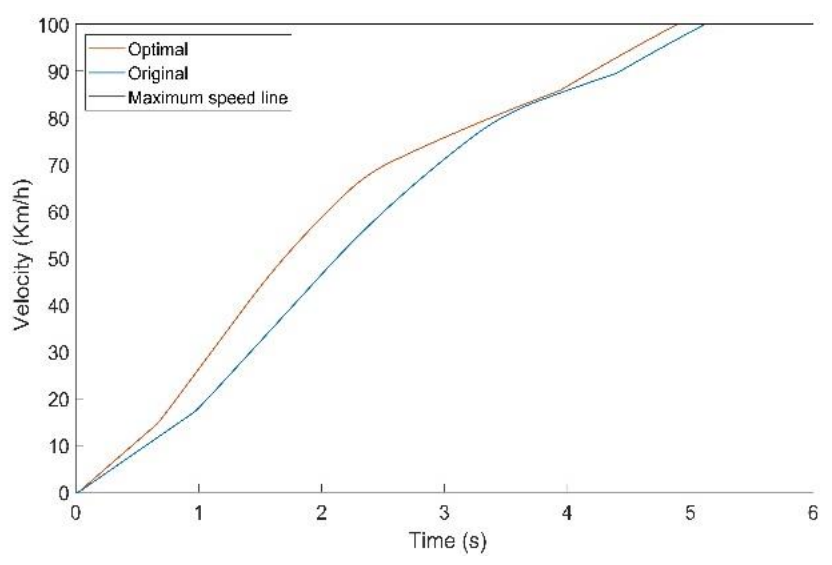

Fig. 9 Acceleration curve

We can see that in the SOC comparison figure, the optimized power consumption is higher than that before optimization, because it can be seen from the economic objective function (1) that power consumption is more cost-effective than fuel consumption. Therefore, on the premise of meeting the driver's torque demand, the consumption of MG2 should be increased as much as possible to improve the economy. 


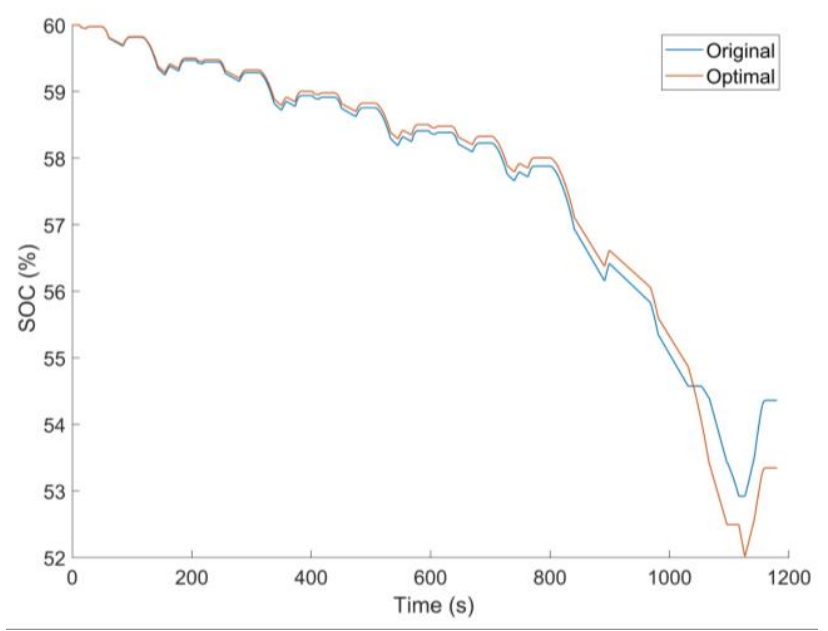

Fig. 10 SOC comparison

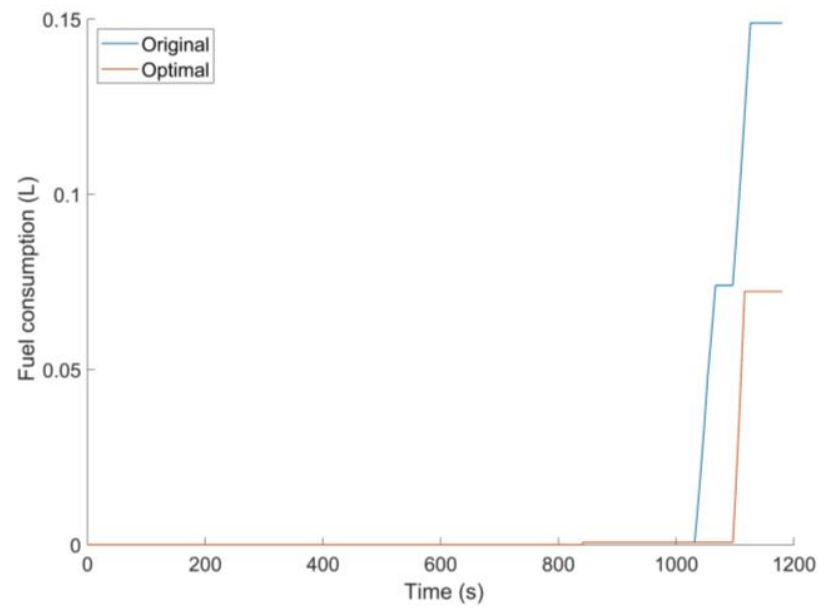

Fig. 11 Fuel consumption comparison

The Fig. 12 shows the comparison of mode transition frequency before and after optimization under the NEDC condition. It is noted that in the dotted line frame, frequent switching of $\mathrm{OEM} \rightarrow \mathrm{ENA} \rightarrow \mathrm{EM} 1 \mathrm{~S} \rightarrow \mathrm{OEM} \rightarrow$ $\mathrm{EM} 1 \mathrm{~S} \rightarrow \mathrm{OEM} \rightarrow$ EM1S $\rightarrow$ ENA appears in the final suburban segment before optimization, and the optimized result significantly reduces the frequency of mode switching, which can effectively improve driving comfort.

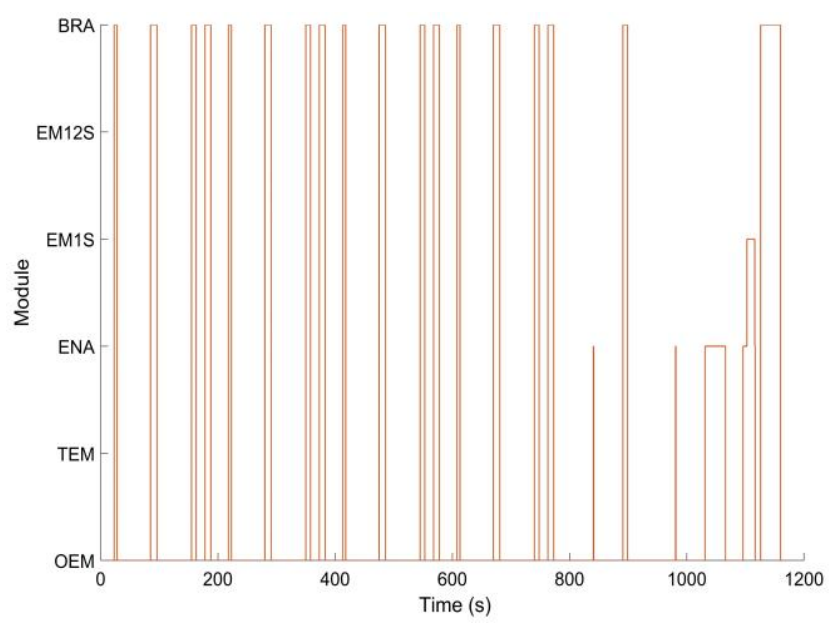

a

Fig. 12 Pattern change

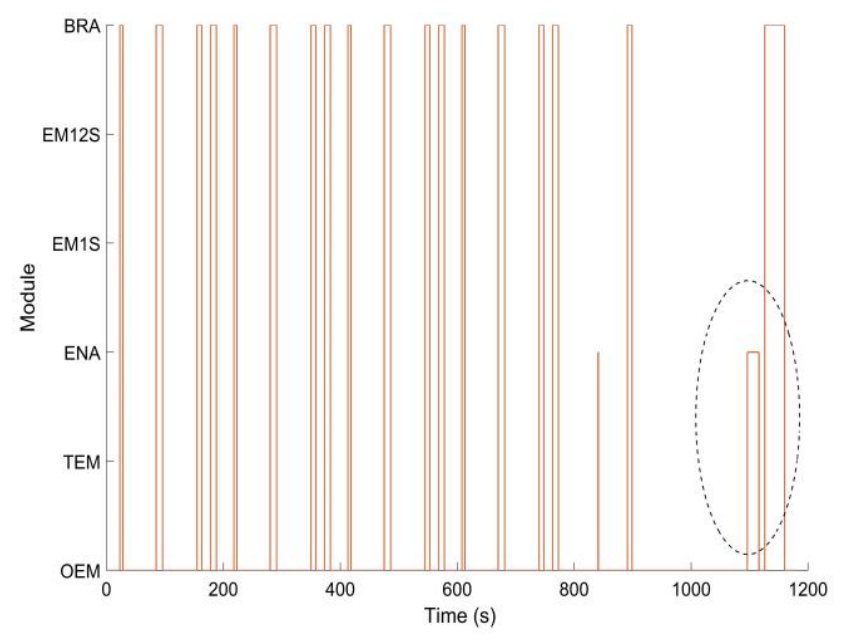

$\mathrm{b}$

Fig.12 Continuation

The comparison between the optimal results and the original results is shown in the following Table 4.

Table 4

Comparison of optimization results

\begin{tabular}{|c|c|c|c|}
\hline Parameter & $\begin{array}{c}\text { Pre-optimization } \\
\text { results }\end{array}$ & $\begin{array}{c}\text { Optimized } \\
\text { results }\end{array}$ & $\begin{array}{c}\text { Optimized } \\
\text { percentage }\end{array}$ \\
\hline$t_{a c c}, \mathrm{~s}$ & 5.146 & 4.865 & $5.5 \%$ \\
\hline$f_{\text {eco }}$, yuan & 1.3138 & 1.11 & $15.5 \%$ \\
\hline$f_{s}, \mathrm{~m} / \mathrm{s}^{3}$ & 0.583 & 0.3643 & $37.5 \%$ \\
\hline$f_{f}, \mathrm{~Hz}$ & 0.0996 & 0.0339 & $65.9 \%$ \\
\hline
\end{tabular}

In order to further verify the generality of the energy management strategy proposed in this paper, the four comprehensive conditions including NEDC, UDDS, FTP and WLTC were used for verification. The comprehensive working conditions are shown in the Fig. 13.

As can be seen from the figure above, the engine start-stop, acceleration and deceleration of the comprehensive working condition are more frequent. The regularity of the vehicle cycle is worse. However, under the EMS and optimized parameters proposed in this paper, good speed tracking can still be maintained. The results show that the optimized vehicle has good dynamic performance.

Through the engine's work points distribution map, under the comprehensive working condition, the optimized engine working points are mainly distributed in low-power areas, and the motor charging is used to ensure that the engine can work in the high-efficiency range as much as possible.

Through the operating points distribution diagram of MG1, it can be seen that the MG1 works less due to the multiple use of the engine before optimization. After optimization, the operating points are concentrated in the middle and high speed, middle and low torque, and the working efficiency can be guaranteed to be high.

And in the operating points distribution diagram (b) of MG2, after optimization, except for part of the working points distributed in the low speed and low torque area caused by the use of the motor during startup, the rest are distributed in the middle and low speed area, which can ensure the efficient operation of MG2.

We can see from the above diagram that under the comprehensive working conditions, the vehicle still focuses 
on power consumption while meeting the power demand, so as to get the best economic performance. Finally, through the calculation of economic objective function, the economic performance is 6.0489 yuan before optimization and 5.0093 yuan after optimization.

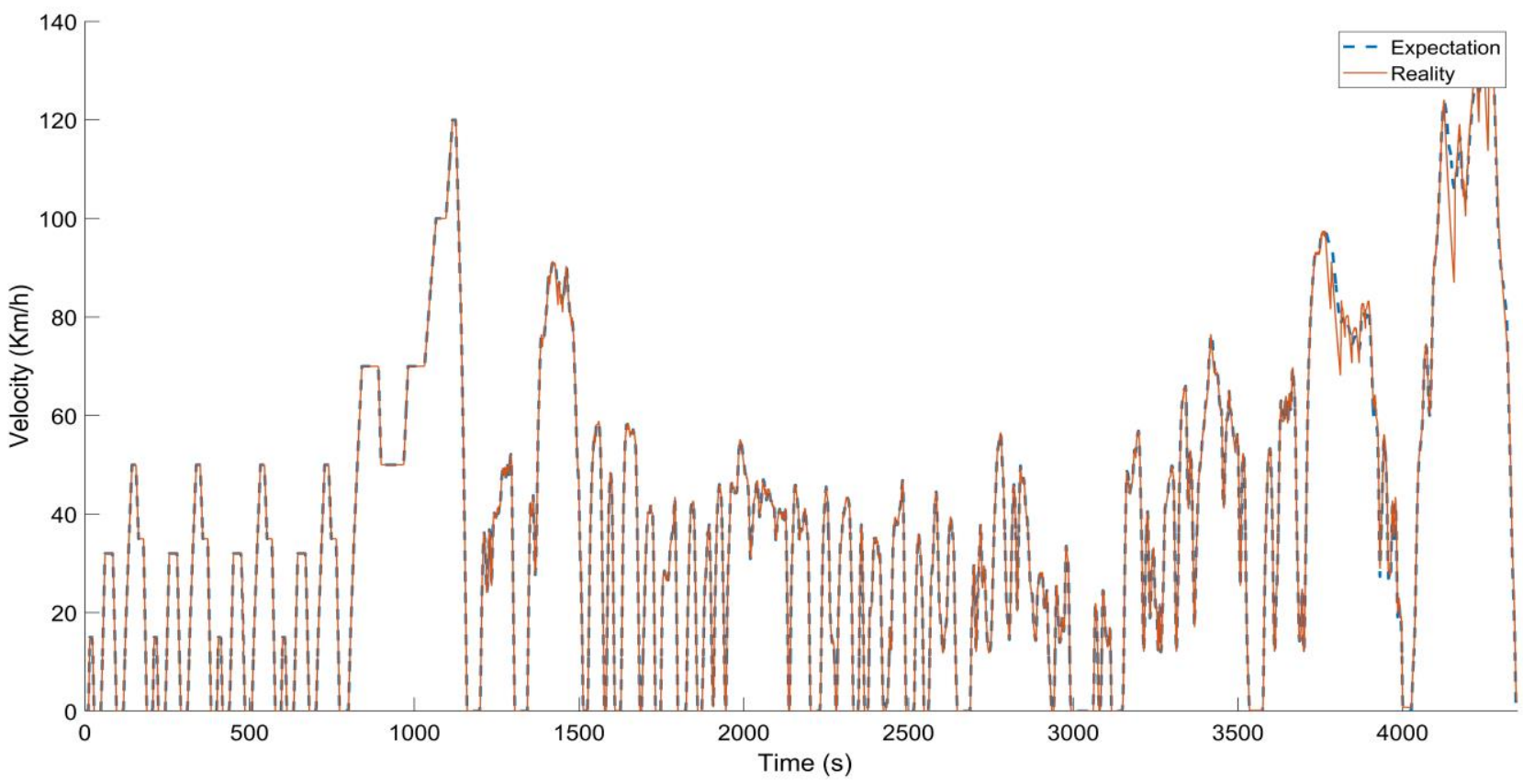

Fig. 13 Speed tracking curve

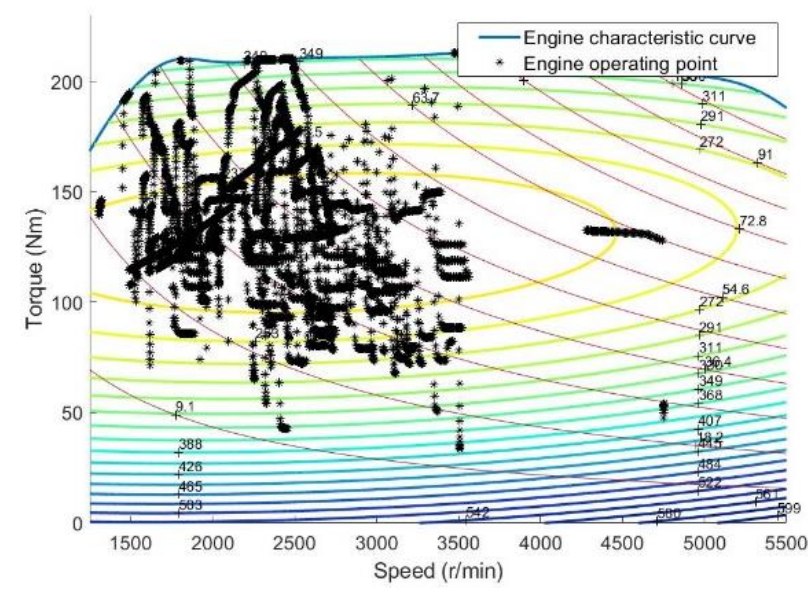

a) before optimization

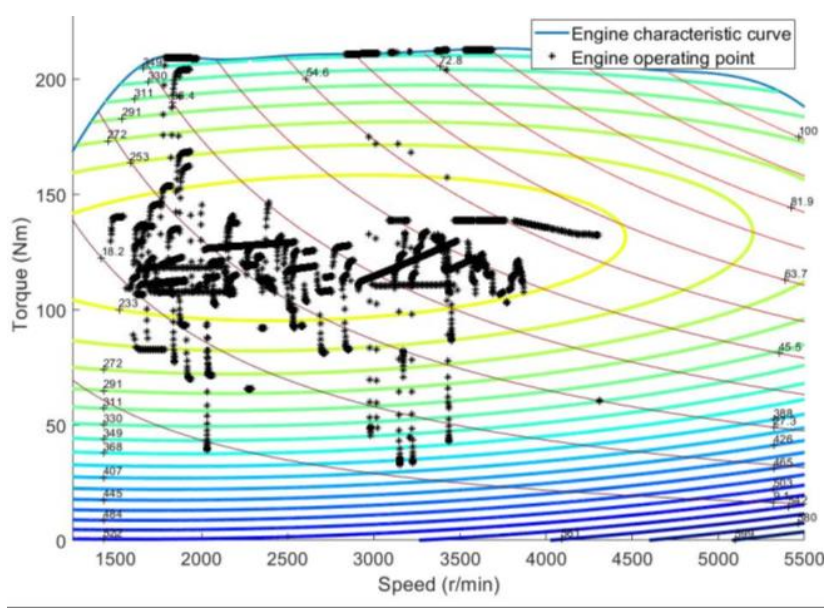

b) after optimization

Fig. 14 Engine operating point distribution

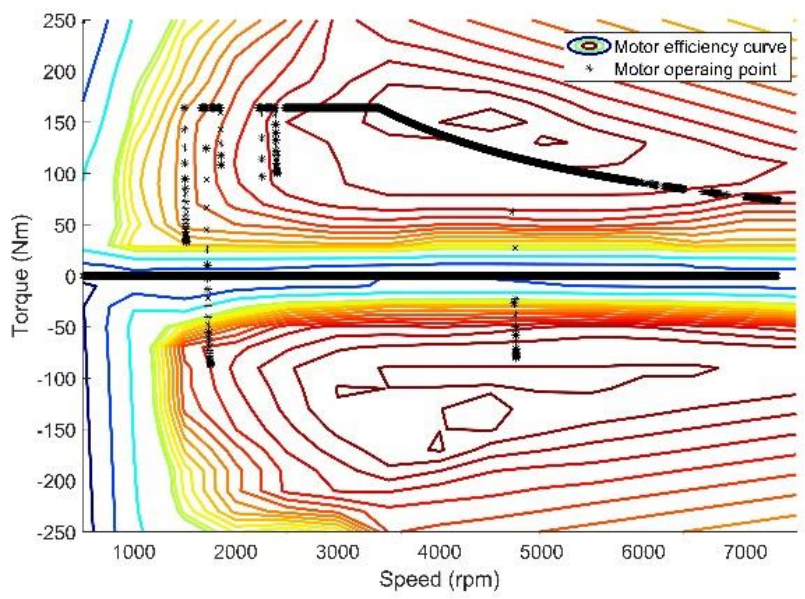

a) before optimization

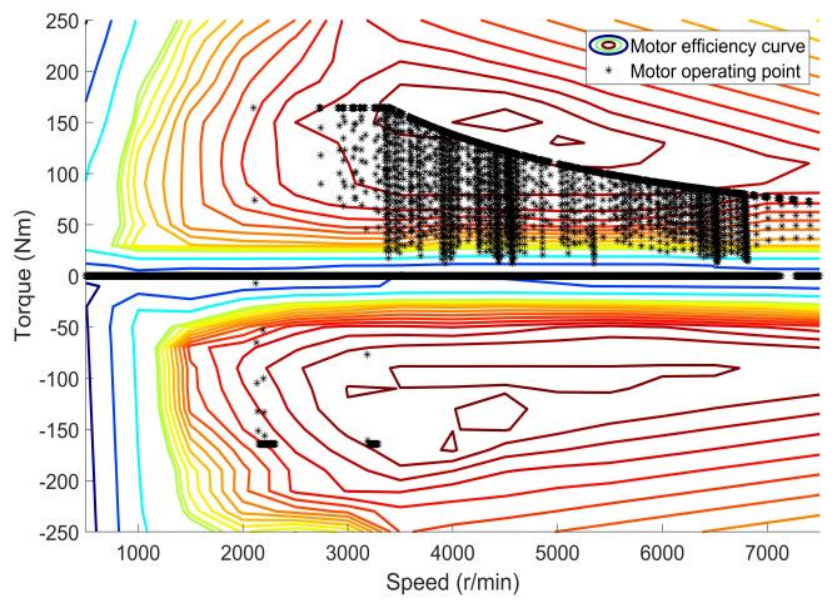

b) after optimization

Fig. 15 MG1 operating point distribution 


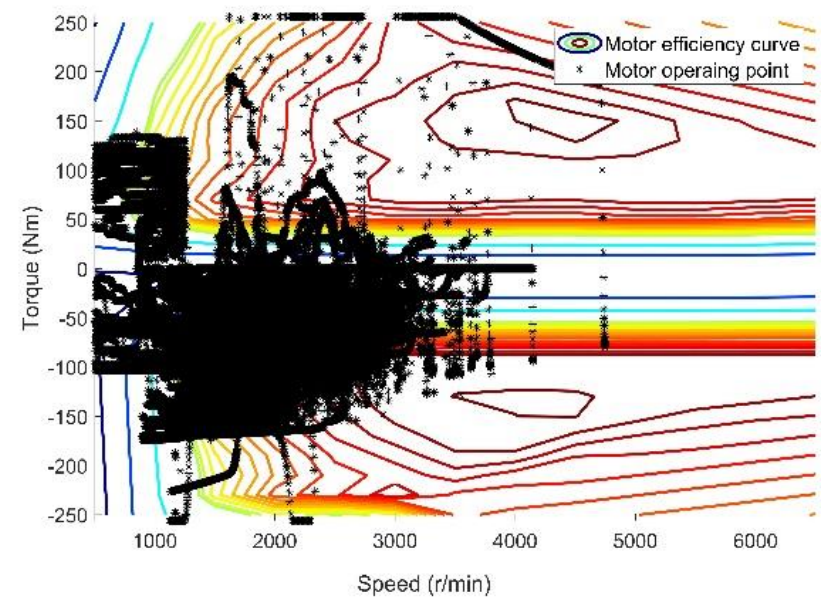

a) before optimization

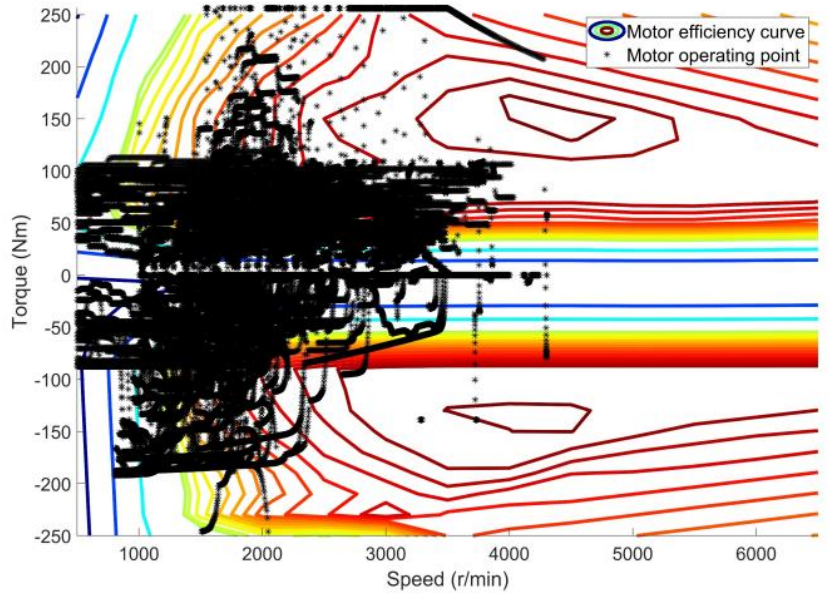

b) after optimization

Fig. $16 \mathrm{MG} 2$ operating point distribution

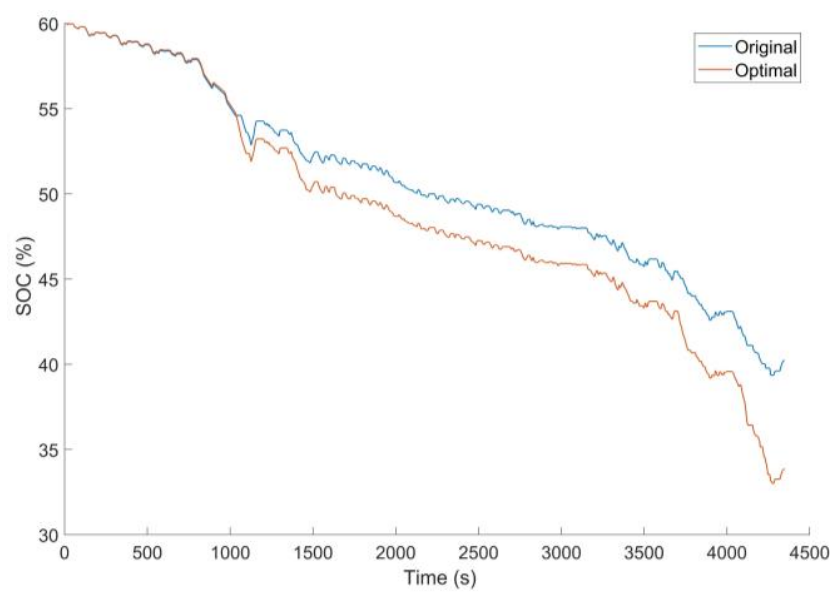

Fig. 17 Comparison of comprehensive SOC

The figure above (a) is the mode switching diagram under the comprehensive working condition before optimization, and (b) is the mode switching diagram after optimization. In the figure, the obvious part of the optimization result is magnified, which is more convenient for observation. It can be seen that under the comprehensive working condition, using the energy management strategy proposed in this paper, the engine start stop frequency and mode switching frequency are obviously reduced.

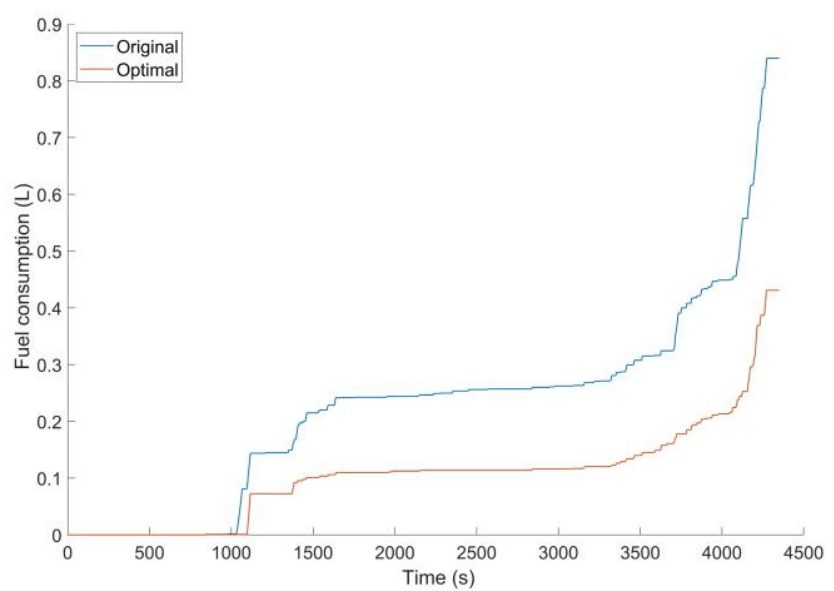

Fig. 18 Comparison of comprehensive fuel consumption

According to the comfort objective function calculation, the mode transition frequency is $0.1253 \mathrm{~Hz}$ before optimization, $0.1101 \mathrm{~Hz}$ after optimization, and the frequency is reduced by $12.1 \%$; Through the calculation of ride smooth objective function, the average positive impact degree is $0.4430 \mathrm{~m} / \mathrm{s}^{3}$, the negative impact degree is $0.2735 \mathrm{~m} / \mathrm{s}^{3}$ before optimization, and the optimized results are $0.4058 \mathrm{~m} / \mathrm{s}^{3}$ and $-0.2604 \mathrm{~m} / \mathrm{s}^{3}$ respectively, $8.4 \%$ and $4.8 \%$ lower, respectively

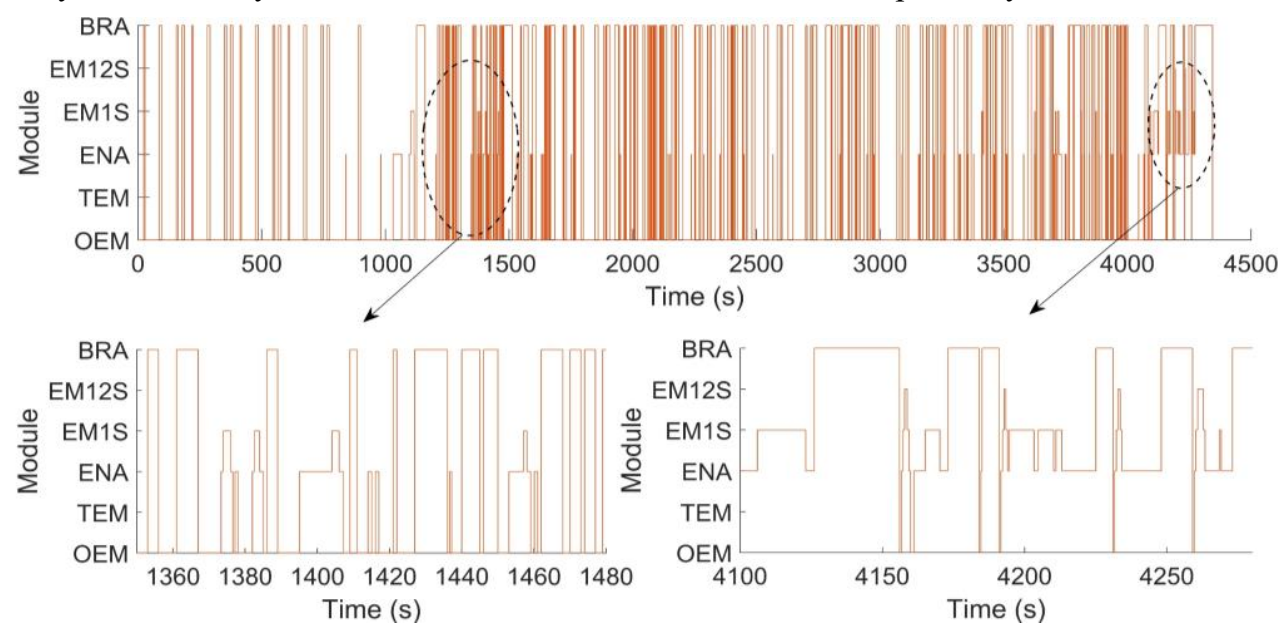

a

Fig. 19 Mode transition under comprehensive conditions 


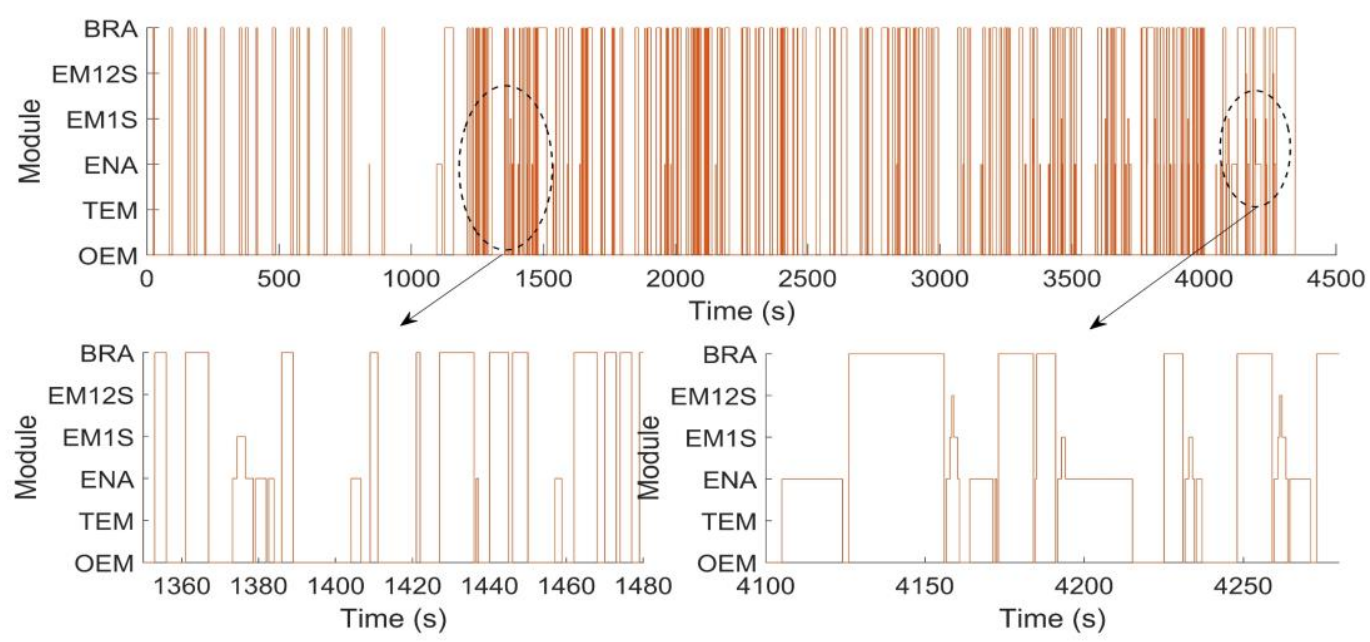

b

Fig. 19 Continuation

Table 5 terest with respect to the research, authorship, and/or publi-

Comparison of optimization results under comprehensive conditions

\begin{tabular}{|c|c|c|c|}
\hline Parameter & $\begin{array}{c}\text { Pre-optimization } \\
\text { results }\end{array}$ & $\begin{array}{c}\text { Optimized } \\
\text { results }\end{array}$ & $\begin{array}{c}\text { Optimized } \\
\text { percentage }\end{array}$ \\
\hline$f_{\text {eco }}$, yuan & 6.0489 & 5.0093 & $17.2 \%$ \\
\hline$f_{s}, \mathrm{~m} / \mathrm{s}^{3}$ & 0.4430 & 0.4058 & $8.4 \%$ \\
\hline$f_{f}, \mathrm{~Hz}$ & 0.1253 & 0.1101 & $12.1 \%$ \\
\hline
\end{tabular}

\section{Conclusion}

In this paper, a multi-objective dynamic optimization method is proposed, compared with the traditional optimization method, this method completes the two-layer real-time dynamic optimization and added a comfort objective function, and smoothness was used as a constraint condition to optimize the logic threshold and the transmission ratio. Completed decoupling the parameter of the driveline and the control strategy, and the final-result can reach the global optimum. Consider the mode transition at the beginning of parameter design, reduce the workload for later parameter matching and calibration, and improve the work efficiency of later vehicle improvement. In the selection of optimization parameters, the threshold of high efficiency working area of engine is considered, which effectively reduces the frequent start and stop of the engine and ensures that the working points fall in the high efficiency range as much as possible. The results show that the multi-objective dynamic optimization method considering mode transition can effectively reduce the mode transition frequency compared with the traditional method. Also effectively improve the dynamic performance, economic performance and comfort performance. This paper is a useful reference for studying the comprehensive performance and mode transition frequency of multi-mode hybrid electric vehicles. It also provides ideas for the optimization of hybrid powertrain parameters.

\section{Declaration of conflicting interests}

The author(s) declared no potential conflicts of in- cation of this article.

\section{Acknowledgement}

This work was supported by National Key R\&D Program of China (2017YFB0103201) and the Fundamental Research Funds for the Central Universities of China (PA2019GDZC0101).

\section{References}

1. Wang, W.; Liu, K.; Yang, C.; Xu, B.; Ma, M. 2021. Cyber physical energy optimization control design for PHEVs based on enhanced firework algorithm, Ieee Transactions on Vehicular Technology 70: 282-291. https://doi.org/10.1109/tvt.2020.3046520.

2. Zhou, S.; Liu, X.; Hua, Y.; Zhou, X.; Yang, S. 2021. Adaptive model parameter identification for lithium-ion batteries based on improved coupling hybrid adaptive particle swarm optimization- simulated annealing method, Journal of Power Sources 482. https://doi.org/10.1016/j.jpowsour.2020.228951.

3. Li, P.; Jiao, X.; Li, Y. 2021. Adaptive real-time energy management control strategy based on fuzzy inference system for plug-in hybrid electric vehicles, Control Engineering Practice 107.

https://doi.org/10.1016/j.conengprac.2020.104703.

4. Bingzhan, Z.; Zhihang, C.; Mi, C.; Murphey, Y.L. 2009. Multi-objective parameter optimization of a series hybrid electric vehicle using evolutionary algorithms, IEEE Vehicle Power and Propulsion Conference 921925. https://doi.org/10.1109/vppc.2009.5289749.

5. Zhang, L.; Hu, X.; Wang, Z.; Sun, F.; Deng, J.; Dorrell, D. G. 2018. Multiobjective optimal sizing of hybrid energy storage system for electric vehicles, Ieee Transactions on Vehicular Technology 67: 1027-1035. https://doi.org/10.1109/tvt.2017.2762368.

6. Song, Z.; Zhang, X.; Li, J.; Hofmann, H.; Ouyang, M.; Du, J. 2018. Component sizing optimization of plug-in hybrid electric vehicles with the hybrid energy 
storage system, Energy 144: 393-403.

https://doi.org/10.1016/j.energy.2017.12.009.

7. Lv, C.; Hu, X.; Sangiovanni-Vincentelli, A.; Li, Y.; Martinez, C. M.; Cao, D. 2019. Driving-style-based codesign optimization of an automated electric vehicle: a cyber-physical system approach, Ieee Transactions on Industrial Electronics 66: 2965-2975.

https://doi.org/10.1109/tie.2018.2850031.

8. Liu, Y.; Liu, Q.; Lv, C.; Zheng, M.; Ji, X. 2018. A Study on objective evaluation of vehicle steering comfort based on driver's electromyogram and movement trajectory, Ieee Transactions on HumanMachine Systems 48: 41-49.

https://doi.org/10.1109/thms.2017.2755469.

9. Chen, Y.; Zhang, Y.; Wei, C. Method for selecting configuration of power system of heavy hybrid electric vehicle, involves completing selection of power system configuration of heavy hybrid electric vehicle based on minimum fuel consumption and emission, Univ. Hebei Technology.

10. Yan, Y.; Li, Q.; Huang, W.; Chen, W. 2021. Operation optimization and control method based on optimal energy and hydrogen consumption for the fuel cell/supercapacitor hybrid tram, Ieee Transactions on Industrial Electronics 68: 1342-1352.

https://doi.org/10.1109/tie.2020.2967720.

11. Peiyang, J.; Xingcheng, W.; Mingyu, C.; Sheng, Y. 2019. Research on an improved equivalent fuel consumption minimization strategy based on ant colony algorithm, Chinese Automation Congress: 2318-2323. https://doi.org/10.1109/cac48633.2019.8996647.

12. Gao, B.; Liang, Q.; Xiang, Y.; Guo, L.; Chen, L. 2015. Gear ratio optimization and shift control of 2-speed IAMT in electric vehicle, Mechanical Systems and Signal Processing 50-51: 615-631. https://doi.org/10.1016/j.ymssp.2014.05.045.

13.Zhang, Y.; Wang, W.; Yang, C.; Han, L.; Zhang, Z.; Liu, J. 2019. An effective regenerative braking strategy based on the combination algorithm of particle swarm optimization and ant colony optimization for electrical vehicle, Ieee 28th International Symposium on Industrial Electronics: 1905-1910. https://doi.org/10.1109/isie.2019.8781183.

14. Li, L.; Zhou, L.; Yang, C.; Xiong, R.; You, S.; Han, Z. 2017. A novel combinatorial optimization algorithm for energy management strategy of plug-in hybrid electric vehicle, Journal of the Franklin Institute-Engineering and Applied Mathematics 354: 6588-6609.

https://doi.org/10.1016/j.jfranklin.2017.08.020.

15. Bingzhan, Z.; Guodong, Z.; Yong, H.; Yaoyao, N.; Mingming, Q. 2020. Optimal energy management for series-parallel hybrid electric city bus based on improved genetic algorithm, Mechanika 26(3):252-259. https://doi.org/10.5755/j01.mech.26.3.2413316.

16. Zhang, P.; Wu, X.; Du, C.; Xu, H.; Wang, H. 2020. Adaptive equivalent consumption minimization strategy for hybrid heavy-duty truck based on driving condition recognition and parameter optimization, Energies 13. https://doi.org/10.3390/en13205407

17. Fang, L.; Qin, S.; Xu, G.; Li, T.; Zhu, K. 2011. Simultaneous optimization for hybrid electric vehicle parameters based on multi-objective genetic algorithms, Energies 4: 532-544. https://doi.org/10.3390/en4030532.

18.Zhou, X.; Qin, D.; Hu, J. 2017. Multi-objective optimization design and performance evaluation for plug-in hybrid electric vehicle powertrains, Applied Energy 208: 1608-1625.

https://doi.org/10.1016/j.apenergy.2017.08.201.

19. Xiaolin, T.; Haitao, Z.; Feng, W.; Weida, W.; Xianke, L. 2021. Longevity-conscious energy management strategy of fuel cell hybrid electric vehicle based on deep reinforcement learning, Energy 238.

https://doi.org/10.1016/J.ENERGY.2021.121593.

20.Kim, H.; Kim, J.; Lee, H. 2011. Mode transition control using disturbance compensation for a parallel hybrid electric vehicle, Proceedings of the Institution of Mechanical Engineers Part D-Journal of Automobile Engineering 225: 150-166.

https://doi.org/10.1243/09544070jauto1523.

21. Lei, Z.; Sun, D.; Liu, Y.; Qin, D.; Zhang, Y.; Yang, Y.; Chen, L. 2017. Analysis and coordinated control of mode transition and shifting for a full hybrid electric vehicle based on dual clutch transmissions, Mechanism and Machine Theory 114: 125-140.

https://doi.org/10.1016/j.mechmachtheory.2017.04.001.

22. Yang, C.; Jiao, X.; Li, L.; Zhang, Y.; Chen, Z. 2018. A robust $\mathrm{H}$-infinity control-based hierarchical mode transition control system for plug-in hybrid electric vehicle, Mechanical Systems and Signal Processing 99: 326-344.

https://doi.org/10.1016/j.ymssp.2017.06.023.

23.Zhang, Y.; Zhao, H.; Huang, K.; Qiu, M.; Geng, L. 2020b. Hybrid optimization and its applications for multi-mode plug-in hybrid electric vehicle, Proceedings of the Institution of Mechanical Engineers Part DJournal of Automobile Engineering 234: 228-244. https://doi.org/10.1177/0954407019837858.

24. Tametang, M. M. I.; Leutcho, G. D.; Yemele, D. 2021. Engine automatic start-stop dynamic analysis and vibration reduction for a two-mode hybrid vehicle, Chaos 31: 073126-073126. https://doi.org/10.1063/5.0057761.

25. Chen, J-S.; Hwang, H-Y. 2013. Engine automatic startstop dynamic analysis and vibration reduction for a twomode hybrid vehicle, Proceedings of the Institution of Mechanical Engineers Part D-Journal of Automobile Engineering 227: 1303-1312.

https://doi.org/10.1177/0954407013491184.

26. Fu, J.; Song, S.; Fu, Z.; Ma, J. 2019. Design of coordinated control strategy during driving mode switching for parallel hybrid electric vehicles, Transactions of the Institute of Measurement and Control 41: 2507-2520. https://doi.org/10.1177/0142331218803669.

27. Ogawa, H.; Takahashi, Y. 2021. Echo state network based model predictive control for active vibration control of hybrid electric vehicle powertrains, Applied Sciences 11:6621-6621. https://doi.org/10.3390/APP11146621.

28.Zuo, Y.; Sun, R.; Zang, J.; Zheng, M. 2020. Coordinated control for driving mode switching of hybrid electric vehicles, Shock and Vibration 2020. https://doi.org/10.1155/2020/7325456. 
K. Huang, Zh. Tang, M. Qiu, D. Wu, B. Zhang

\section{RESEARCH ON MULTI-OBJECTIVE PARAMETER DYNAMIC OPTIMIZATION DESIGN OF HEV CON- SIDERING MODE TRANSITION}

\section{S u m m a r y}

The parameter optimization of hybrid electric vehicle is very complex and has always been the focus of research, due to the highly intertwined relationship between powertrain configurations and EMS. In addition, automated powertrain is typical cyber-physics system that contains discrete states, such as modes and gears. Transition between discrete states is a contributing factor that causes discomfort. Therefore, in order to solve the above problems, this paper proposes a multi-objective dynamic optimization algorithm considering mode transition. The algorithm consists of two layers. The first layer optimizes fuel economy and dynamic performance, the second layer optimizes ride comfort and smoothness performance, and the results of the second layer are returned to the first layer for re-optimization in real time. The mode transition frequency is used as the comfort objective function, and the threshold value of the engine high efficiency working area is added to the optimization parameter selection. The results show that the optimization method proposed in this paper realizes the parameter decoupling, and the optimization results achieve the global optimization. Effectively improves the vehicle performance and reduces engine start-stop frequently.

Keywords: multi-objective dynamic optimization; decouple; mode transition; ride comfort; fuel economy.

Received June 06, 2021

Accepted February 04, 2022

This article is an Open Access article distributed under the terms and conditions of the Creative Commons Attribution 4.0 (CC BY 4.0) License (http://creativecommons.org/licenses/by/4.0/). 